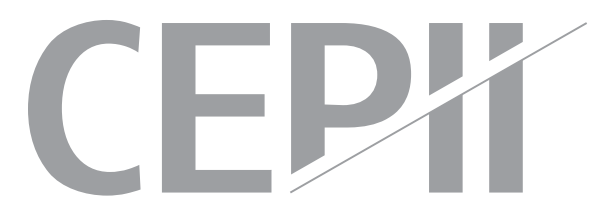

\title{
Fiscal Consolidations and Public Debt in Europe
}

Gianluca Cafiso \& Roberto Cellini

\section{Highlights}

- In recent times a wide debate has developed in Europe concerning the effect of fiscal consolidations on the evolution of the public-debt/GDP ratio.

- In the context of the current crisis, fiscal consolidations were meant to reduce the debt/GDP ratio. However, the restrictive effect of a fiscal consolidation on the GDP might well offset the deficit reduction and cause an undesired debt/GDP increase.

- Past events suggest a positive short-term effect, while the most-relevant medium-term effect seems to be negative. On the whole, savings-based fiscal consolidations are to be preferred in any case. 


\section{Abstract}

The objective of this paper is to gain insights into the relationship between deficit-reducing policies and the evolution of the debt/GDP ratio. We consider past events of fiscal consolidation in a selected group of EU countries and check what is the associated change of the debt/GDP ratio both from a short and medium-term perspective. As for the medium-term perspective, we do also differentiate between tax-based and savings-based fiscal consolidations. Our results point towards a positive short-term effect, while the medium-term effect turns out to be negative. Savingsbased fiscal consolidations result to be less negative on the debt/GDP ratio's evolution than tax-based ones.

\section{Keywords}

Fiscal consolidations, debt/GDP ratio, Europe.

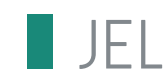

H63, E63. 


\title{
Fiscal Consolidations and Public Debt in Europe
}

\author{
Gianluca Cafiso \& Roberto Cellini ${ }^{+}$
}

\section{Introduction}

In recent times a wide debate has developed concerning the effects of restrictive fiscal policies on the dynamics of the public debt/GDP ratio (DGR). The debate is nourished by the current experience of EU countries, where fiscal consolidations have been implemented with the objective of reducing their DGR. An objective which is now made mandatory by the new Six-pack and Fiscal Compact agreements which impose a precise reduction path for the countries exceeding the $60 \%$ Maastricht threshold.

The object of this paper is the study of how the DGR dynamics changed when a fiscal consolidation was implemented with the intent to reduce the overall deficit. From an economic-policy perspective, building on past episodes, we aim to provide information helping to assess whether fiscal authorities' effort to contain the DGR through fiscal consolidations will be effective or not. Indeed, the restrictive effect of a fiscal consolidation on the GDP might well offset the deficit reduction and cause an undesired DGR increase. As a matter of fact, this would be a self-defeating outcome which cannot be excluded ex-ante (Gros 2011, Krugman 2011, Sutherland et al. 2012) and which has a clear theoretical reference in the fiscal multiplier literature (see, among the others, Cwik \& Wieland 2011 for their focus on the Euro Area).

Our analysis covers a selected group of EU countries observed over the period 1980-2009. We have two research objectives. First, we want to assess how the DGR has changed in the past when a fiscal consolidation was implemented. We do this taking both a short and medium-term perspective to allow for the possibility of a varying fiscal multiplier (Clinton et al. 2011, Coenen et al. 2008, Corsetti et al. 2012). For reasons which we explain in the text, we investigate this through a Comparison of Distributions approach. Second, we want to check whether differences in the DGR evolution emerge when fiscal consolidations are either taxbased or savings-based. We study this for the medium-term effect, the most relevant from a policy-perspective, through a Logistic Estimation which is also functional to test the robustness of the results found.

\footnotetext{
${ }^{\times}$Corresponding Author. University of Catania and CESifo. E-mail: gcafiso@unict.it, Webpage: www.sites.google.com/site/giancafiso.

+ University of Catania. E-mail: cellini@unict.it; Webpage: www.robertocellini.it. Acknowledgments

We wish to thank Andreas Steiner for his excellent discussion of this paper at the $69^{\text {th }}$ IIPF Conference (Taormina, August 2013). Gratitude goes also to Jacopo Cimadomo for preliminary talks about this topic and suggestions.

Gianluca Cafiso thanks the CEPII (Paris-France) for his research visiting there in June and July 2013. His contribution to this paper was accomplished during that period, gratitude goes to the CEPII staff for useful comments.
} 
This work flows into a research stream devoted to the understanding of how fiscal policy affects macroeconomic variables (Alesina \& Ardagna 2010, Guajardo et al. 2011) and which explicitly considers different policy options in the presence of public debt (Corsetti et al. 2011). It is particularly relevant in the assessment of the actions taken to tackle the Euro Area debt crisis started in 2010 (Cafiso 2012a). Our contribution to the literature consists in the use of a more reliable series of fiscal-consolidation events for the study of their effect on the evolution of the DGR, in the consideration of a possibly different effect across the short and medium-term, and in the distinction between tax and savings-based fiscal consolidations for the study of their effect on the DGR.

The paper is structured as follows. Section 2 introduces to the budget relations which are object of our empirical investigation. Section 3 describes the data set of fiscal-consolidation events used. Section 4 outlines the analysis. In section 5 we discuss the short and mediumterm results. Section 6 reports the output of the logistic estimation. We draw the conclusions of our analysis in section 7. In Appendix I we cluster the countries into two groups to detect those which are more likely to suffer an adverse evolution of the DGR in case of a fiscal consolidation, in Appendix II we briefly discuss the case of consecutive fiscal consolidations.

\section{Budget Relations: a simple algebra of deficit and debt dynamics}

The budget relations at the basis of our empirical investigation can be easily derived from national budget accounting (Escolano 2010), we present them in this section in a way inspired by Gros (2011). The difference equation which describes the debt evolution is:

$B_{t}=O D_{t}+B_{t-1}$

where $B_{t}$ is the debt level and $O D_{t}$ is the overall deficit, such that $O D_{t}=P D_{t}+i_{t} B_{t-1} ; P D_{t}$ is the primary deficit and $i_{t} B_{t-1}$ is the interest bill. Since $O D_{t}$ is the overall deficit (and not the balance), $\Delta O D_{t}>0$ indicates a deficit increase. Dividing eq. (1) for the nominal GDP $\left(Y_{t}\right)$ and considering that $\mathrm{Y}_{\mathrm{t}-1} / \mathrm{Y}_{\mathrm{t}}=1 /\left(1+g_{t}\right)$, we get:

$$
\frac{B_{t}}{Y_{t}}=\frac{O D_{t}}{Y_{t}}+\frac{B_{t-1}}{Y_{t-1}} \frac{1}{\left(1+g_{t}\right)}
$$

\footnotetext{
1 The analysis here considers the overall deficit; for a discussion of how differently the primary balance and the interest bill determine the debt evolution, refer to Cafiso (2012b).
} 
Using small letters for GDP ratios and re-expressing the equation in terms of first differences, we obtain:

$\Delta b_{t} \stackrel{\text { def }}{=} b_{t}-b_{t-1}=o d_{t}+\frac{b_{t-1}}{1+g_{t}}-b_{t-1}$

By using eq. (3), we now show the possible effects of a deficit reduction on the DGR variation both in the short-term and over a longer period. The distinction between the short and a longer period is done in accordance with the fiscal multiplier literature (among the others, Clinton et al. 2011, Coenen et al. 2008, Corsetti et al. 2012) which explicitly distinguishes between the short and medium term effect of fiscal policy on the GDP.

The following discussion develops considering the DGR variation, not the DGR level. Indeed, as it will be made clear in section 4.3, we are interested in its average change in fiscalconsolidation years with respect to years of no fiscal-consolidation. ${ }^{2}$

\section{Short-term effect of a deficit reduction on the DGR}

Gros (2011) defines the short-term effect of a deficit reduction on the DGR as the contemporaneous DGR variation caused by such deficit reduction; in symbols: $\Delta o d_{t}<0 \rightarrow \Delta b_{t}$. This effect can be quantified through the derivative $\partial \Delta b_{t} / \partial o d_{t}$ of eq. (3) when $g_{t}=g\left(o d_{t}\right)$ is assumed:

$$
\frac{\partial \Delta b_{t}}{\partial o d_{t}}=1-\frac{b_{t-1}}{\left(1+g_{t}\right)^{2}} \cdot \frac{\partial g_{t}}{\partial o d_{t}}
$$

Notice that $\partial \Delta b_{t} / \partial o d_{t}>0$ means that a higher (lower) deficit will cause a higher (lower) debt variation. Then, a lower deficit will not affect positively the same-year DGR when the opposite holds true, that is $\partial \Delta b_{t} / \partial o d_{t}<0$ (self-defeating outcome):

$\frac{\partial \Delta b_{t}}{\partial o d_{t}}<0 \Rightarrow 1<\frac{\partial g_{t}}{\partial o d_{t}} \cdot\left[b_{t-1} \cdot\left(1+g_{t}\right)^{-2}\right]$

$\partial g / \partial o d$ resembles the fiscal multiplier. When it is equal to +1 and $\left(1+g_{t}\right)^{-2} \cong 1$, condition (5) holds for a country with a DGR larger than one. Clearly, even in this simple framework, the outcome of the consolidation depends upon the size of the fiscal multiplier.

\footnotetext{
${ }^{2}$ For this reason, the derivatives derived in section 2.1 and 2.2 are defined for the DGR variation. However, they are formally equal to those for the DGR level.
} 
Real-world debt and deficit data do not allow to analyse effectively the intra-year effect $\left(\Delta o d_{t}<0 \rightarrow \Delta b_{t}\right)$ in empirical studies. Then, to study the short-term effect, we will compare the DGR variation contemporaneous to a fiscal consolidation with the variation recorded in the previous two years; this will serve to check changes in the DGR trend.

\section{Longer-term effect of a deficit reduction on the DGR}

The longer-term effect of a deficit reduction is defined as the cumulated $m$-periods DGR variation caused by a year $t$ deficit reduction; in symbols: $\Delta o d_{t}<0 \rightarrow \Delta_{m} b_{t+m-1}$, where $\Delta_{m} b_{t+m-1}=b_{t+m-1}-b_{t-1}$. Gros (2011) considers the longer-term effect of a deficit reduction in two alternative cases, namely under a temporary or definitive deficit reduction.

I) Temporary Deficit Reduction: $\Delta o d_{t}<0$ and $\Delta o d_{t+1}>0$ such that $o d_{t-1}=o d_{t+1}$.

Several sub-cases for different GDP reactions are possible, let us consider some of them: 1a) no output drop, $Y_{t-1}=Y_{t}=Y_{t+1}$; 1b) temporary output drop, $Y_{t-1}>Y_{t}$ and $Y_{t}<Y_{t+1}$ such that $\left.Y_{t-1}=Y_{t+1} ; 1 c\right)$ permanent output drop, $Y_{t-1}>Y_{t}=Y_{t+1}=\ldots=Y_{t+\infty}$.

In case $1 a$ the maximum longer-term effect of a temporary deficit reduction materializes because less debt has been added through less deficit in $t$ at a constant output level. As for case $1 b$, the longer-term effect is positive, if and only if, the output drop in $t$ does not offset completely the deficit reduction. Case $1 c$ is unlikely to happen since the deficit reduction under scrutiny is temporary.

II) Definitive Deficit Reduction: $\Delta o d_{t}<0$ and $o d_{t}=\ldots=o d_{t+m-1}$.

Also in this case, some sub-cases are to take into account: 2a) no output drop, $Y_{t-1}=Y_{t}=Y_{t+1}$; 2b) temporary output drop, $Y_{t-1}>Y_{t}$ and $Y_{t}<Y_{t+1}$ such that $Y_{t-1}=Y_{t+1}$; 2c) permanent output drop, $\left.\quad Y_{t-1}>Y_{t}=Y_{t+1}=\ldots=Y_{t+\infty} ; 2 d\right)$ prolonged output drop, $Y_{t-1}>Y_{t}=Y_{t+1}=\ldots=Y_{t+m-1}$ but $Y_{t-1}=Y_{t+m}$.

In case $2 a$ the cumulative beneficial effect is to take for-given, because less debt has been added through comparatively less deficit in each year at a constant GDP level. As for case $2 b$, a beneficial effect in terms of DGR evolution is to be expected from $t+1$ onwards. Again, case $2 c$ is not likely because the output tends to return to its growth path in the long-run even in case of a permanent deficit reduction.

\footnotetext{
${ }^{3}$ The size of the multiplier is a debated issue in the literature because, among other things, it determines whether or not fiscal stimuli are worth to invert a recession. For a recent discussion of this issue referring to the policy answer to the 2008-2009 crisis, see Cwick \& Wieland (2011).
} 
Case $2 d$ needs more consideration and it is relevant for the following analysis. We develop the discussion here by considering a 2-year time horizon $(m=2)$. Since we apply such 2-year horizon also in the following empirical analysis, we will talk of medium-term effects of fiscal consolidations in the empirical part. In section 4.2 we explain why it makes sense to consider a 2-year horizon.

Now, let us imagine that the deficit reduction keeps down the GDP level for $m=2$, then we need to evaluate: $o d_{t-1}>o d_{t}=o d_{t+1} \rightarrow \Delta_{2} b_{t+1}$ under the hypothesis $Y_{t-1}>Y_{t}=Y_{t+1}$. Starting from eq. (1) translated in $t+1$, after simple manipulations we obtain:

$$
\frac{B_{t+1}}{Y_{t+1}}=\frac{O D_{t+1}}{Y_{t+1}}+\left[\frac{O D_{t}}{Y_{t}} \frac{1_{t}}{\left(1+g_{t+1}\right)}+\frac{B_{t-1}}{Y_{t-1}} \frac{Y_{t-1}}{Y_{t}} \frac{Y_{t}}{Y_{t+1}}\right]
$$

By considering the conditions defining case $2 d$, namely $Y_{t-1}>Y_{t}=Y_{t+1}$, equation (6) in terms of 2-year cumulated change and GDP-ratios becomes:

$$
\Delta_{2} b_{t+1} \stackrel{\text { def }}{=} b_{t+1}-b_{t-1}=o d_{t+1}+\left[o d_{t}+\frac{b_{t-1}}{1+g_{t}}\right]-b_{t-1}
$$

Taking into account that $o d_{t}=o d_{t+1}$ under case $2 d$, the effect of a permanent deficit reduction may be expressed as the first derivative $\partial \Delta_{2} b_{t+1} / \partial o d_{t}$ of eq. (7):

$$
\left.\frac{\partial \Delta_{2} b_{t+1}}{\partial o d_{t}}\right|_{o d_{t}=o d_{t+1}}=1+1-b_{t-1} \cdot\left(1+g_{t}\right)^{-2} \cdot \frac{\partial g_{t}}{\partial o d_{t}} \cong 2-b_{t-1} \cdot \frac{\partial g_{t}}{\partial o d_{t}}
$$

From equation (8), the condition for a self-defeating outcome is:

$$
\left.\frac{\partial \Delta_{2} b_{t+1}}{\partial o d_{t}}\right|_{o d_{t}=o d_{t+1}}<0 \Rightarrow 2<b_{t-1} \cdot \frac{\partial g_{t}}{\partial o d_{t}}
$$

If the fiscal multiplier is constant across the short and longer-term, condition (9) is more difficult to meet than condition (5) because the product of the fiscal multiplier and the starting debt/GDP ratio must now exceed 2 .

However, in the real world, the fiscal multiplier is not likely to be constant (Clinton et al. 2011, Coenen et al. 2008, Corsetti et al. 2012). Then, there is no reason why the short and longerterm effect of a fiscal consolidation on the DGR should be of the same sing. This is the very reason why we distinguish between the short and longer-term effect of fiscal consolidations in this study of their impact on the DGR. 


\section{About Fiscal Consolidations data}

To develop our analysis we need data on Fiscal Consolidations (FCs). There are three main methods to compile series of FC events and quantify their budget effect. The first consists in calculating significant variations of the Cyclically-Adjusted Primary Balance (CAPB approach, Alesina \& Ardagna 2010). The second relies on identification based on the estimation of a structural VAR (Ramey 2013). The third consists in the so-called Narrative Approach (Romer \& Romer 2010).

With reference to the CAPB method used in Alesina \& Ardagna (2010), a paper close to ours for the objective of their analysis, Devries et al. (2011) explain that there are several problems with it. In particular, cyclical adjustment methods do not offset the effect of assets price variations linked to the output dynamics (imperfect cyclical adjustment bias). As a consequence, the significant variations detected can still be due to the output fluctuation and not to discretionary policies.

To overcome this, Devries et al. (2011) adopt the narrative approach to compile a reliable series of FC events. By considering national official documents, they detect those years when a fiscal consolidation was enforced with the explicit intent to reduce the budget deficit. Then, they compile their data set by including the budget effect of those fiscal consolidations (in GDP terms) as reported in the same documents. This method ensures that the events considered are those when the government really set in place measures to reduce the deficit. The authors claim that, by doing so, there cannot be years erroneously considered of fiscal consolidation as a drawback of a mechanic procedure as the CAPB approach. Guajardo et al. (2011), almost the same authors as in Devries et al. (2011), apply such new data set to test the expansionary austerity hypothesis as documented in several contributions (Giavazzi \& Pagano 1990, among the others). With a specific reference to Alesina \& Ardagna (2010), their objective is to prove that such hypothesis emerges as a bias of using significant variation of the CAPB to account for changes in the fiscal stance. They provide evidence that this is the case by showing that fiscal consolidations cause real GDP contractions. ${ }^{4}$

Perotti (2013) discusses in details both the CAPB and the narrative approach. He explains that data sets of this kind are generally affected by the countercyclical response bias and by the imperfect cyclical adjustment bias. He explains that the narrative approach avoids the latter but not the former, while the CAPB is flawed by both. Then, he concludes that data sets such as Devries et al. (2011) are an improvement. Indeed, that data set has been used more and more in empirical studies (among many others, Alesina et al. 2013).

After careful consideration of all these points, we decided to resort to the Devries et al. (2011)'s data set as well to account for FC events. We believe that such choice improves the reliability of our analysis with respect to previous ones, such as Alesina \& Ardagna (2010),

\footnotetext{
${ }^{4}$ The results in Guajardo et al. (2011) are relevant for our analysis because the negative effect of fiscal consolidations on the GDP affects the association between fiscal consolidations and the DGR, as discussed in section 2 .
} 
which detect fiscal consolidations through the CAPB method when studying their impact on the DGR.

\section{Outline of the Analysis}

In this section we provide information about the empirical analysis developed. We take a first look at the data used for the analysis (the budget effect of fiscal consolidations and the debt/GDP ratio) and at their relationship through descriptive statistics. Then, we introduce the two variables (both based on variations of the DGR) used to study the short and mediumterm effect. In the last subsection we motivate the analytical approach taken.

\subsection{The BEC and the DGR series}

Our sample consists of yearly observations for thirteen EU countries available in Devries et al. (2011)'s data set (Austria, Belgium, Denmark, Finland, France, Germany, Ireland, Italy, the Netherlands, Portugal, Spain, Sweden, the United Kingdom) over the 1980-2009 period.

To account for fiscal consolidations, we build the variable BEC which consists of 390 observations (balanced panel, with 13 countries observed over a 30 year period). For every country $i$, year $t$ may be a fiscal consolidation year (yes-FC) or not (no-FC). The variable $B E C$ is set equal to zero in case of no-FC, while in case of yes-FC it is equal to the Budget Effect of the Fiscal Consolidation (BEFC, in terms of GDP) as reported in Devries et al. (2011):

$B E C=\left\{\begin{array}{c}B E F C \text { if } t \text { is a yes-FC year } \\ 0 \text { if } t \text { is a no-FC year }\end{array}\right.$

In case of yes-FC, the distribution of $B E C$ is very heterogeneous among countries, as shown in Table 1-Panel X. The largest BEC values pertain to the cases of Ireland in 2009 (4.74\%), Italy in 1993 (4.49\%), Italy in 1995 (4.20\%), and Finland in 1993 (3.71\%).

The distribution of the DGR variations $(\Delta b)$ is reported in Table 1 under different conditions. In Panel A we report the distribution of the whole sample. Positive variations are more than negatives $\left(50^{\text {th }}\right.$ percentile is positive) and the bulk of values lies to the left of the mean (positive skewness): DGR increases have been more frequent than decreases. In our analysis we compare the distribution of DGR variations under different policy stances. Accordingly, if one considers years of yes-FC against years of no-FC (panel $C$ versus panel $B$ ), it emerges that negative DGR variations (favourable evolution) are by far more in no-FC

\footnotetext{
${ }^{5}$ We ourselves have compared the data set by Devries et al. (2011) with what obtained considering variations of the CAPB; we used CAPB data made available by the EU commission (Ameco database). As matter of fact, the two series do not diverge excessively. The main problem consists in setting the significance threshold for CAPB variations. This is why the narrative approach a-là Devries et al. (2011) seems more reliable in the end.
} 
years than in yes-FC years. Moreover, the larger the BEC, the smaller the portion of negative DGR variations (comparison of median values across panel $\mathrm{D}$ and $\mathrm{E}$ ).

Table 1. Distribution of BEC and $\Delta b$ in different cases

\begin{tabular}{|c|c|c|c|}
\hline \multicolumn{2}{|c|}{ Panel X: BEC distr. in case of yes-FC (obs 120) } & \multicolumn{2}{|c|}{ Panel A: $\Delta b$, whole sample (obs 367 ) } \\
\hline Descriptive statistics & Percentiles & Descriptive statistics & Percentiles \\
\hline $\begin{array}{l}\text { Mean } 1.210 \\
\text { Std. Dev. } 1.031 \\
\text { Skewness } 1.060\end{array}$ & $\begin{array}{l}25 \%: .500 \\
50 \%: 1.025 \\
75 \%: 1.655\end{array}$ & $\begin{array}{l}\text { Mean .907 } \\
\text { Std. Dev. } 4.798 \\
\text { Skewness } .835\end{array}$ & $\begin{array}{l}25 \%:-2.233 \\
50 \%: .400 \\
75 \%: 3.299\end{array}$ \\
\hline \multicolumn{2}{|c|}{ Panel B: $\Delta b$, no-FC (obs 257) } & \multicolumn{2}{|c|}{ Panel C: $\Delta b$, yes-FC (obs 110$)$} \\
\hline Descriptive statistics & Percentiles & Descriptive statistics & Percentiles \\
\hline $\begin{array}{l}\text { Mean .376 } \\
\text { Std. Dev. } 4.792 \\
\text { Skewness } .717\end{array}$ & $\begin{array}{l}25 \%:-2.698 \\
50 \%: .100 \\
75 \%: 2.703\end{array}$ & $\begin{array}{l}\text { Mean } 2.147 \\
\text { Std. Dev. } 4.596 \\
\text { Skewness } 1.345\end{array}$ & $\begin{array}{l}25 \%:-.651 \\
50 \%: 1.254 \\
75 \%: 3.770\end{array}$ \\
\hline \multicolumn{2}{|c|}{ Panel D: $\Delta b$, yes-FC \& BEC>0 (obs 102) } & \multicolumn{2}{|c|}{ Panel E: $\Delta b$, yes-FC \& $B E C>\mathrm{p} 50$ th (obs 53 ) } \\
\hline Descriptive statistics & Percentiles & Descriptive statistics & Percentiles \\
\hline $\begin{array}{l}\text { Mean } 2.334 \\
\text { Std. Dev. } 4.635 \\
\text { Skewness } 1.402\end{array}$ & $\begin{array}{l}25 \%:-.651 \\
50 \%: 1.350 \\
75 \%: 4.100\end{array}$ & $\begin{array}{l}\text { Mean } 3.359 \\
\text { Std. Dev. } 5.095 \\
\text { Skewness } 1.073\end{array}$ & $\begin{array}{l}25 \%: .300 \\
50 \%: 2.516 \\
75 \%: 6.100\end{array}$ \\
\hline
\end{tabular}

Notes: $\cdot \Delta b$ is the variation of the DGR. $\cdot$ The DGR data are from the ECFIN Ameco database.

Correlation values are in Table 2 - Panel A. The correlation between $B E C_{t}$ and $\Delta b_{t}$ is positive: FC events are associated with higher contemporaneous DGR variations. The correlation remains quite high also between $B E C_{t}$ and $\Delta b_{t+1}$, but it is negligible between $B E C_{t}$ and $\Delta b_{t+2}$. Their association therefore seems to last only 1-period ahead, for this reason we use a 2-year horizon in our analysis of the medium-term effect.

The correlation between $B E C$ and the real-GDP growth rate $(r g)$ is negative: the larger the fiscal consolidation, the smaller $r$. The correlation between real-GDP growth and the DGR variation is strongly negative too: the smaller the GDP growth, the larger the government's financing needs. Furthermore, the average real-GDP growth in yes-FC years is below than in the full sample and in no-FC years (all values in Table 2 - Panel B). 
Table 2. Pairwise correlations and average Real GDP growth rates in different cases

\begin{tabular}{|c|c|c|c|c|c|c|c|}
\hline \multicolumn{8}{|c|}{ Panel A: Pairwise correlations between variables of interest } \\
\hline & $B E C_{t}$ & $\Delta b_{t}$ & $\Delta b_{t+1}$ & $\Delta b_{t+2}$ & $r g$ & $n g$ & $B g$ \\
\hline$B E C_{t}$ & 1.000 & & & & & & \\
\hline$\Delta b_{t}$ & .316 & 1.000 & & & & & \\
\hline$\Delta b_{t+1}$ & .126 & .609 & 1.000 & & & & \\
\hline$\Delta b_{t+2}$ & -.022 & .301 & .608 & 1.000 & & & \\
\hline $\mathrm{rg}$ & -.166 & -.590 & -.468 & -280 & 1.000 & & \\
\hline$n g$ & -.063 & -.044 & .019 & .058 & .423 & 1.000 & \\
\hline $\mathrm{Bg}$ & .185 & .832 & .560 & .274 & -.373 & .365 & 1.000 \\
\hline \multicolumn{8}{|c|}{ Panel B: Real GDP growth rates under different cases } \\
\hline & & Case & Mean & Stad. Dev. & Freq. & & \\
\hline & & yes-FC & 2.073 & 2.171 & 259 & & \\
\hline & & no-FC & 2.406 & 2.605 & 118 & & \\
\hline & & whole & 2.301 & 2.480 & 377 & & \\
\hline
\end{tabular}

Notes: $-\Delta b$ is the variation of the DGR; $r g$ is the growth rate of real GDP; $n g$ is the growth rate of nominal GDP; $B g$ is the growth rate of the stock of debt. - Apart for the BEC series, all the data are from the ECFIN Ameco database.

\subsection{Variables for the Short and Medium-Term effects}

In this section we introduce the two variables used to analyse the short and the medium-term effects of fiscal consolidations (FCs) on the DGR evolution.

\section{Short-term: Trend-Break}

To study the short-term effect of a FC on the DGR evolution we construct the $D T b_{i t}$ variable which we call Trend-Break. It accounts for deviations of the current DGR variation $\left(\Delta b_{t}\right)$ with respect to the average DGR variation in the previous two years ( $t-1$ and $t-2)$ :

$D T b_{i t}=\left(\frac{\Delta b_{i t-1}+\Delta b_{i t-2}}{2}\right)-\Delta b_{i t}$

where $\Delta b_{t}=b_{t}-b_{t-1}$ and $b_{t}$ is the debt/GDP ratio (DGR).

$D T b_{i t}>0$ signals a favourable evolution in year $t$ : the current increase in $b$ is smaller than the average increase recorded in the two previous years, or the current DGR decrease is larger with respect to the previous 2-year average. $D T b_{i t}<0$ signals an adverse evolution in year $t$ : either the DGR increase is higher, or its reduction smaller, with respect to the previous 2-year average. 
We focus our analysis on non-marginal deviations by considering only $D T b_{i t}$ above the $25^{\text {th }}$ percentile of each country absolute-value distribution. Then, we consider only $D T b_{i t}{ }^{*}$ in what follows:

$D T b_{i t}^{*}=\left\{\begin{array}{c}D T b_{i t} \text { if } D T b_{i t} \geq 25^{\text {th }} \text { percentile of abs }\left(D T b_{i t}\right) \\ 0 \text { if } D T b_{i t}<25^{\text {th }} \text { percentile of abs }\left(D T b_{i t}\right)\end{array}\right.$

Then, $D T b_{i t}{ }^{*}$ can be either positive, negative or zero in any year $t$. We judge a fiscal consolidation favourable on the DGR short-term evolution, if we observe a positive $D T b_{i t}{ }^{*}$ value (favourable outcome). The by-country plot of $D T b_{i t}{ }^{*}$ is in Figure 1.

Figure 1. Trend-Break variable, by Country

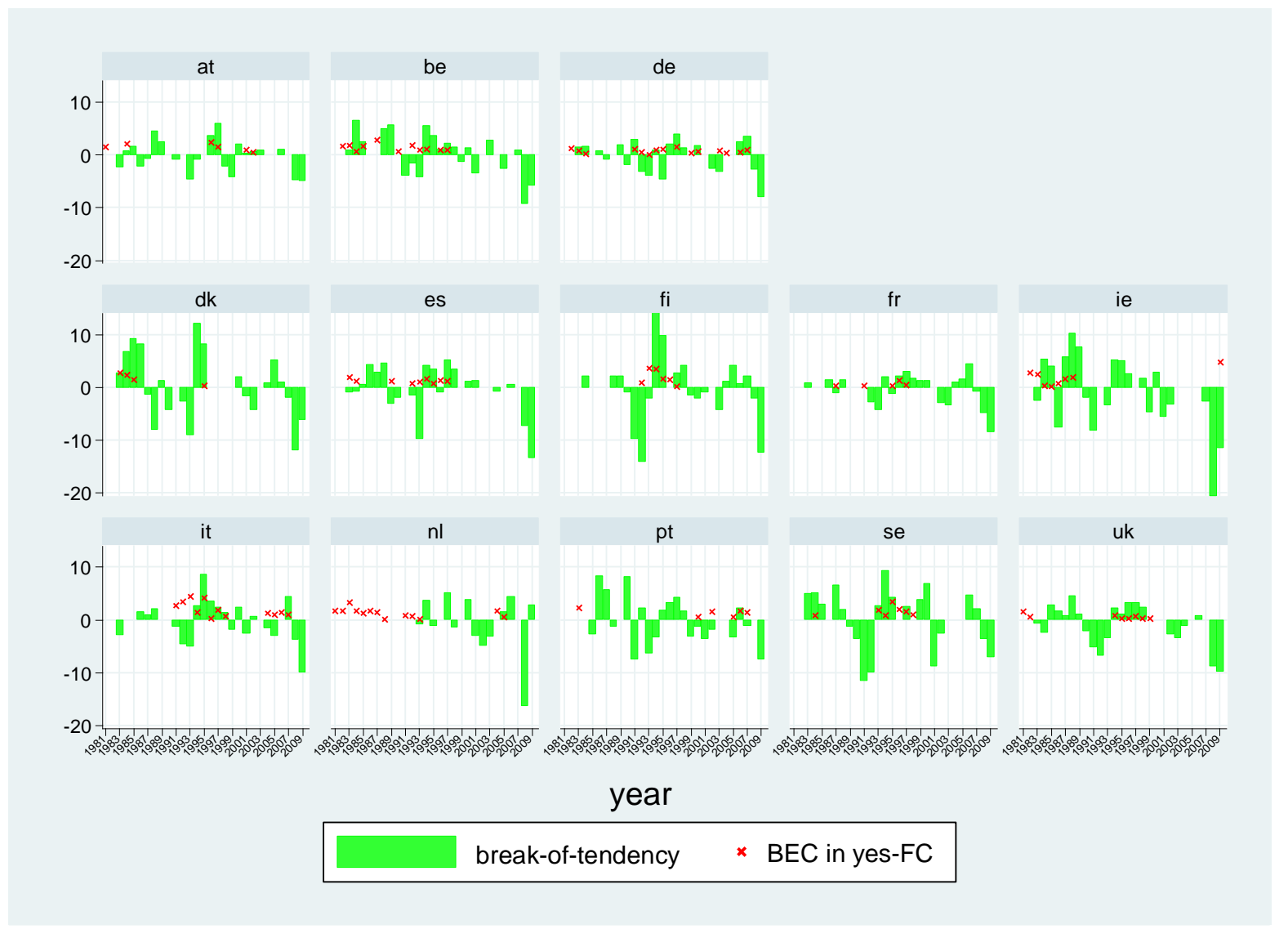




\section{Medium-Term: Cumulated Change}

We study the medium-term effect of fiscal consolidations on the DGR by considering the 2year cumulated change:

$$
\Delta_{2} b_{i t+1}=\Delta b_{i t+1}+\Delta b_{i t}+=b_{i t+1}-b_{i t-1}
$$

where $\Delta b_{t}=b_{t}-b_{t-1}$ and $b_{t}$ is the DGR. As already mentioned, we opt for a 2-year horizon because correlations in Table 2 signal a significant effect up to the following year only. ${ }^{6}$

As for the variable $D T b_{i t}$, we focus on non-marginal cumulated changes. We therefore consider only $\Delta_{2} b_{i t+1}$ values above the $25^{\text {th }}$ percentile of country is absolute-value distribution:

$\Delta_{2} b_{i t+1}^{*}=\left\{\begin{array}{c}\Delta_{2} b_{i t+1} \text { if } \Delta_{2} b_{i t+1} \geq 25^{\text {th }} \text { percentile of abs }\left(\Delta_{2} b_{i t+1}\right) \\ 0 \text { if } \Delta_{2} b_{i t+1}<25^{\text {th }} \text { percentile of abs }\left(\Delta_{2} b_{i t+1}\right)\end{array}\right.$

In every year $t, \Delta_{2} b_{i t+1}^{*}$ can be positive, negative or non-significant (equal to zero). We judge a fiscal consolidation favourable on the DGR medium-term evolution if we observe an associated negative $\Delta_{2} b_{i t+1}^{*}$ (favourable outcome). The by-country plot of the $\Delta_{2} b_{i t+1}^{*}$ variable is in Figure 2.

In Appendix I $\Delta_{2} b_{i t+1}$ is also used for a cluster analysis which splits the country sample into two groups in order to understand among which countries similarities in the DGR evolution emerge.

\footnotetext{
${ }^{6}$ Other authors choose a 3-year period instead. We tested our conclusions also using such horizon, and they remain largely unchanged.
} 


\section{Figure 2. Cumulated Change, by Country}

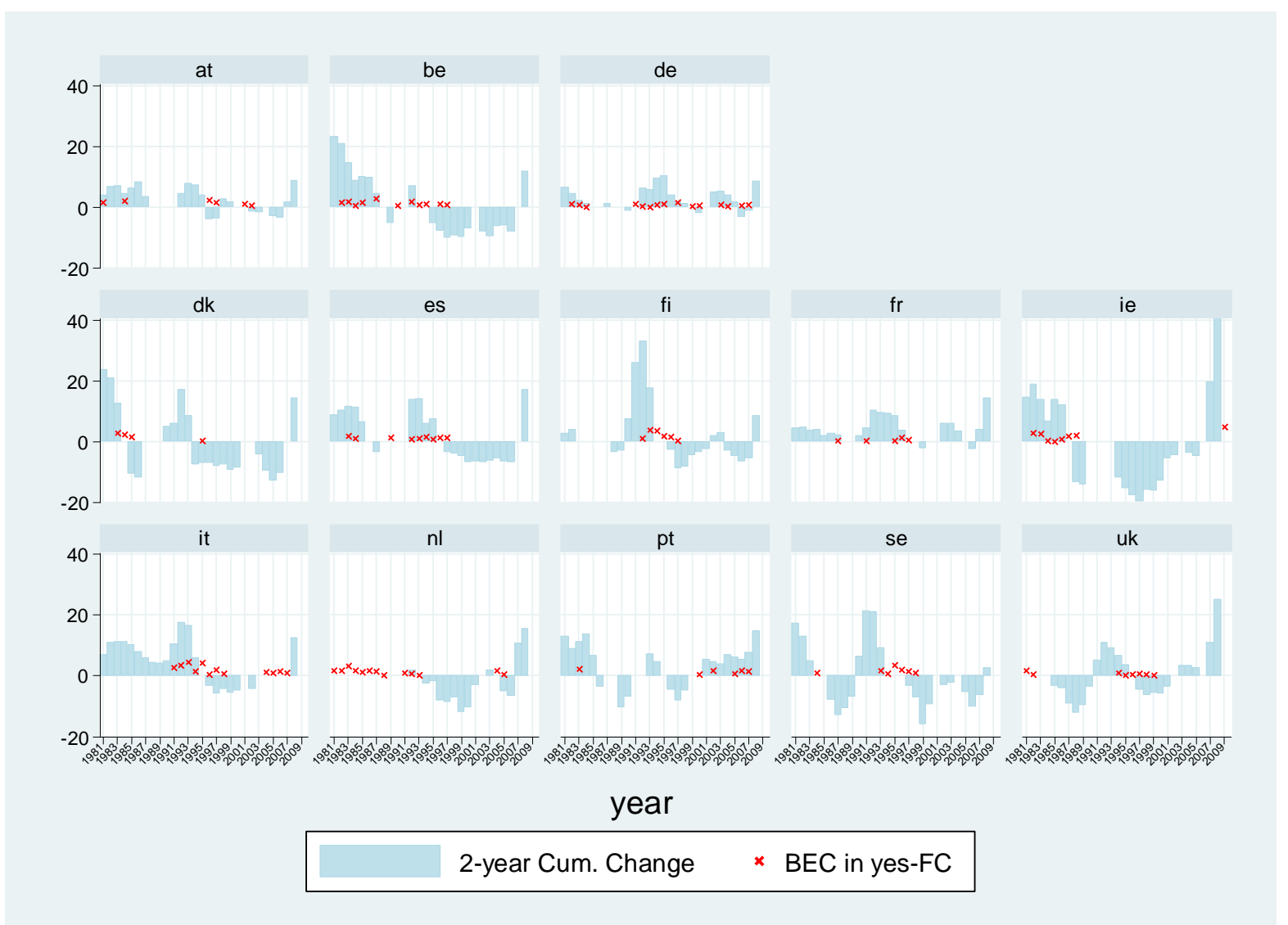

\subsection{Motivations behind the Analytical Approach taken}

As it is clear from eq. (3), the object of our analysis is the relationship between two variables related to the government budget identity. Then, these are simultaneous to each other by construction, with their deviation resulting from the sign and magnitude of the fiscal multiplier. Furthermore, fiscal consolidations can be either the cause or the consequence of a certain DGR evolution. To wit, we cannot exclude that the discretionary fiscal consolidations reported in Devries et al. (2011) were enforced to limit the public debt expansion. Then, the decision of a fiscal consolidation cannot be assumed exogenous with respect to the DGR evolution: this implies a reverse-causality issue.

\footnotetext{
7 Likely, in more recent years and for the Euro Area countries under considerations, lower growth is the cause of deficitreducing consolidations because of externally-imposed discipline such as the Stability and Growth pact (since 1999). However, further in the past, EU countries committed often to deficit-reducing measures for other reasons too. Just to provide an example here, in 1984 Belgium lunched a three year programme to reduce its deficit (1984-1987). The 1984 IMF Recent Economic Developments reports that it was motivated by "the awareness that the borrowing requirement
} 
Simultaneity and reverse causality represent good reasons to opt for a non-econometric approach when one wants to look at the relationship between fiscal consolidations and the DGR evolution (Alesina \& Ardagna 2010). Accordingly, as a first move, we prefer an approach based on the comparison of distributions which we believe to be more robust to the above-mentioned issues. Such approach, which we apply in section 5, is inspired by Alesina \& Ardagna (2010). However, they limit themselves to compare means without testing their statistical difference. Differently, we compare the frequency of the favourable outcome (through ratios) under different policy stances and evaluate the statistical significance of its variation through non-parametric tests. In this regard, we therefore improve with respect to previous contributions.

In section 6, we develop a regression analysis as a robustness check of the medium-term effect and for further investigation. The regression is a logistic one and it is aimed to estimate the probability of the favourable outcome. The transformation of the dependent variable (from continuous to dichotomous) and the consideration of probabilities are likely to soften the above-mentioned problems. In the end, we will consider the output of the logistic regression in comparison with that of the distribution approach, and vice versa, in order to draw robust conclusions.

The regression analysis will regard the medium-term effect only because it is the more policy-relevant. Indeed, apart for the research interest in the difference between the short and medium-term effect, it is the cumulated change which matters from a policy-perspective (to wit, sustainability).

\section{Comparison of Distributions: Short and Medium-Term effects}

In this section we develop the first part of our analysis to understand how the DGR has changed when a fiscal consolidation was implemented. For the reasons explained in the introduction and in section 2, we check this considering both the short and medium-term through the trend-break and cumulated change variables.

\section{Short-term: Trend-Break}

Our goal is to check whether a discontinuity in the DGR evolution emerges (present with respect to the past) when a fiscal consolidation is enforced. This is achieved through the analysis of the distribution of the $D T b_{i t}^{*}$ variable, a favourable outcome is observed in case of $D T b_{i t}^{*}>0$. The output of the analysis is in Table 3.

In the whole sample (266 observations, both yes-FC and no-FC years), the share of positive values of $D T b_{\text {it }}{ }^{*}$ is $53.0 \%$ (id A in Table 3). Then, positive and negative deviations are almost

was approaching a self-perpetuating level through the 'snowball effect' on interest payments". For a detailed discussion of the background of each FC event included in our sample, see Devries et al. (2011). 
equal in number. If we consider yes-FC years against no-FC years, such ratios diverge: $64.8 \%$ positive in case of yes-FC (id C), $47.2 \%$ positive in case of no-FC (id B).

We test whether such ratios are statistically different from each other, through the Chisquared Test (Conover 1999) which checks association, and the Rank-sum Test (Wilcoxon 1945) which checks origin from the same distribution. Both tests reject the null hypothesis (p.B=p.C, in Table 3) and signal that the distribution of positive $D T b_{i t}{ }^{*}$ is statistically different under the two policy stances. This is also supported by the output of the Binomial Probability Tests which indicates that the share of positive $D T b_{\text {it }}{ }^{*}$ in case of no-FC is statistically equal to half ( $p . B=0.5$ in Table 3), while it is not in case of yes-FC ( $p . C=0.5)$.

These results suggest that fiscal consolidations are associated with a larger portion of favourable outcomes. This finding meets desired expectations about the effect of fiscal consolidations intended to correct the debt evolution. Nonetheless, it is worth emphasising that the portion of favourable outcomes is anyway far from what one might desire (to wit, closer to $100 \%)$.

We do also search possible threshold effects regarding the distribution of the $D T b_{i t}{ }^{*}$ variable in yes-FC years. For fiscal consolidations with a budget effect above the median of the BEC distribution (id D), the ratio of favourable outcomes does not change much. This is also the case when we restrict the observation to $D T b_{i t}{ }^{*}$ values corresponding to a DGR level above $100 \%$ GDP (id E). On the whole, threshold-effects do not seem to be present.

Table 3. Trend-Break analysis

\begin{tabular}{|c|c|c|c|}
\hline \multicolumn{4}{|c|}{ Panel A: Distribution of observations } \\
\hline & Total number of $D T b^{*}{ }_{i t}$ & Share of positive $D T b^{*}{ }_{i t}$ & ID code for test \\
\hline All obs & 266 & $53.0 \%$ & $\mathrm{~A}$ \\
\hline No-FC & 178 & $47.2 \%$ & B \\
\hline Yes-FC & 88 & $64.8 \%$ & C \\
\hline Yes-FC, $>50^{\text {th }} \mathrm{pc}$ & 43 & $62.3 \%$ & $\mathrm{D}$ \\
\hline Yes-FC, DGR>100\% & 21 & $66.7 \%$ & $E$ \\
\hline \multicolumn{4}{|l|}{ Panel B: Tests } \\
\hline & Comparison concerning & $\mathrm{HO}$ & Result - p statistics \\
\hline Association test & $\mathrm{B}, \mathrm{C}$ & p.B=p.C & $\mathrm{P}=.007$ ( $\mathrm{H} 0$ rejected $)$ \\
\hline Rank-sum test & $B, C$ & p.B=p.C & $\mathrm{P}=.000$ (H0 rejected) \\
\hline Binomial probability & $\begin{array}{l}A \\
B \\
C\end{array}$ & $\begin{array}{l}\text { p.A }=.5 \\
\text { p. } B=.5 \\
\text { p.C }=.5\end{array}$ & $\begin{array}{l}\mathrm{P}=.358 \text { (H0 not-rejected) } \\
\mathrm{P}=.500 \text { (H0 not-rejected) } \\
\mathrm{P}=.007 \text { (H0 rejected) }\end{array}$ \\
\hline
\end{tabular}

Note: Association test is the Conover (1999) Chi-squared Test which checks association, the Rank-sum test is the Wilcoxon (1945) test which checks origin from the same distribution, the Binomial probability test (Stata 2009) checks the likelihood of a specified probability. H0: $p . B=p . C$ means that the null hypothesis is the equality of portions in case B and in case $C$. The null hypothesis is rejected or not rejected at the $5 \%$ significance level. 


\section{Medium-Term: Cumulated Change}

The short-term effect regards the association between fiscal consolidations and the contemporaneous DGR evolution considered with respect to its past. We now switch to the 2-year cumulated DGR change $\left(\Delta_{2} b_{i t+1}^{*}\right)$ after a fiscal consolidation. A favourable outcome is observed in case of a negative $\Delta_{2} b_{i t+1}^{*}$ (the DGR decreases).

When we consider the whole sample, the ratio of negative $\Delta_{2} b_{i t+1}^{*}$ over the total number (266 obs) is $44.7 \%$ (id $A$ in Table 4): negative 2-year cumulated changes are slightly less than positive ones. Differently, when we compare no-FC years against yes-FC years, ratios diverge: $49.7 \%$ in case of no-FC and $32.4 \%$ in case of yes-FC (id B, C) with their difference being statistically-significant both using the Chi-square and Rank-sum test (p.B=p.C). Also in this case, these results are supported by the output of Binomial Distribution Tests. As for the presence of threshold effects, the ratio of negative $\Delta_{2} b_{i t+1}^{*}$ decreases further with the size of the fiscal consolidation (id $D$ versus id $C$ ), but it is not very sensible to the level of the DGR (id E).

These are bad news as to the effectiveness of fiscal consolidations: FC events are mainly associated with an adverse DGR evolution in the current and following year. Furthermore, the tighter the fiscal consolidation is, the more likely an adverse DGR evolution gets.

Table 4. Cumulated-Change analysis, all countries

\begin{tabular}{|c|c|c|c|}
\hline \multicolumn{4}{|c|}{ Panel A: Distribution of Observations } \\
\hline & Total number of $\Delta_{2} b_{i t}^{*}$ & Share of negative $\Delta_{2} b_{i t}^{*}$ & ID code for test \\
\hline All obs & 266 & $44.7 \%$ & A \\
\hline No-FC & 189 & $49.7 \%$ & B \\
\hline Yes-FC & 77 & $32.5 \%$ & $\mathrm{C}$ \\
\hline Yes-FC, BEC>50thpc & 36 & $22.2 \%$ & $\mathrm{D}$ \\
\hline Yes-FC, DGR>100\% & 16 & $37.5 \%$ & $E$ \\
\hline \multicolumn{4}{|l|}{ Panel B: Tests } \\
\hline & Comparison concerning: & $\mathrm{HO}$ & Result - p statistics \\
\hline Association test & $\mathrm{B}, \mathrm{C}$ & p.B=p.C & $\mathrm{P}=.010(\mathrm{H} 0$ rejected $)$ \\
\hline Rank-sum test & $\mathrm{B}, \mathrm{C}$ & p.B=p.C & $\mathrm{P}=.004$ (H0 rejected $)$ \\
\hline Binomial probability & $\begin{array}{l}\text { A } \\
B \\
C\end{array}$ & $\begin{array}{l}\text { p.A }=.5 \\
\text { p. } B=.5 \\
\text { p.C }=.5\end{array}$ & $\begin{array}{l}\mathrm{P}=.097 \text { (H0 not-rejected) } \\
\mathrm{P}=1.00 \text { (H0 not-rejected) } \\
\mathrm{P}=.002 \text { (H0 rejected) }\end{array}$ \\
\hline
\end{tabular}

Note: Association test is the Conover (1999) Chi-squared Test which checks association, the Rank-sum test is the Wilcoxon (1945) test which checks origin from the same distribution, the Binomial probability test (Stata 2009) checks the likelihood of a specified probability. $\mathrm{H}_{0}$ is rejected or not rejected at the $5 \%$ significance level. 


\section{The Probability of a favourable Medium-Term effect}

An alternative way to check the effect of fiscal consolidations on the DGR evolution is to study how those influence the probability of observing a DGR reduction (favourable outcome). This perspective has been first taken by Nickel et al. (2010). Accordingly, we use a logistic model to estimate the probability of observing major 2-year cumulated DGR reductions. Our goals are: i) to assess whether fiscal consolidations increase the probability of observing DGR reductions; ii) to check whether a difference emerges between tax-based and savings-based consolidations; (iii) to have a robustness check of the medium-term effect studied in section 5 .

The dichotomous variable $Y_{i t}$ is built for the purpose of this analysis using major 2-year cumulated DGR variations $\left(\Delta_{2} b_{i t+1}^{*}\right)$ as defined in section 4.2. Since a fiscal consolidation is judged favourable on the DGR evolution when an associated negative $\Delta_{2} b_{i t+1}^{*}$ is observed, the dependent dichotomous variable $Y_{i t}$ is:

$Y_{i t}= \begin{cases}1 \text { if } \Delta_{2} b_{i, t+1}^{*}<0 \\ 0 \text { if } \Delta_{2} b_{i, t+1}^{*} \geq 0\end{cases}$

The logistic probability model based on $Y_{i t}$ is therefore defined as: $P_{i t}=E\left[Y_{i t}=1 \mid \vec{X}_{i t}\right]=e^{\bar{X}_{i t}} /\left(1+e^{\bar{X}_{i t}}\right)$, where $E\left[Y_{i, t}=1 \mid \vec{X}_{i, t}\right]$ is the conditional expectation of observing a major debt reduction $\left(\Delta_{2} b_{i t+1}^{*}<0\right)$ given the $\vec{X}_{i t}$ vector of explicative variables. Following Nickel et al. (2010) for the selection of the explicative variables, the linear specification estimated is:

$\ln \left[P_{i t} /\left(1-P_{i t}\right)\right]=\alpha+\beta_{1} B E C_{i t}+\beta_{2} h p G D P g a p_{i t}+\beta_{3} I B_{i t}+\varepsilon_{i t}$

where $B E C_{i t}$ includes the budget effect of the fiscal consolidations (section 4.1), $h p G D P g a p_{i t}$ is the GDP gap with respect to the Hodrick-Prescott calculated GDP trend, and $I B_{i t}$ is the Interest Bill. ${ }^{8}$ The estimation output is in Table $5-$ column $1{ }^{9}$

\footnotetext{
8 Nickel et al. (2010) include the Hodrick-Prescot GDP trend as well; we prefer to keep it out, because it is never significant when included, and, differently from the Interest Bill which is also always not-significant, the information provided is already given by the hpGDPgap regressor.

In the two regressions discussed in this section, we apply a pooled-estimation approach. This is for two reasons: first, in the estimation of the overall effect (Table 5 - Column 1), as well as of the differential effect (Table 5 - Column 2), there is not enough within-group variability of the dependent variable and of the categorical variable which classifies the kind of adjustment; second, the conversion of regression coefficients into marginal effects becomes arbitrary when fixedeffects are included because these cannot be taken into account when calculating the empirical probability function.
} 
Table 5. Logistic Estimation of the Total and Differential effect

\begin{tabular}{|c|c|c|c|c|}
\hline & \multicolumn{2}{|c|}{1 - Total Effect } & \multicolumn{2}{|c|}{2 - Differential Effect } \\
\hline \multicolumn{5}{|l|}{ Estimation Output } \\
\hline & coefficients & stand. error & coefficients & stand. error \\
\hline hpGDPgap & $-9.477^{* *}$ & $(3.823)$ & $-9.406^{* *}$ & (3.793) \\
\hline ib & -0.019 & $(0.055)$ & -0.030 & $(0.055)$ \\
\hline BEC (1-tax) & $-0.541^{* *}$ & $(0.209)$ & $-1.784^{* *}$ & $(0.598)$ \\
\hline adj_type $=2-$ mixed & & & $1.426^{* *}$ & $(0.639)$ \\
\hline adj_type = 3-savings & & & $1.240^{*}$ & $(0.647)$ \\
\hline const. & -0.374 & $(0.434)$ & -0.318 & $(0.437)$ \\
\hline No. of obs & 324 & & 324 & \\
\hline No. of $\Delta_{2} b_{i t+1}^{*}<0$ & 120 & & 120 & \\
\hline Wald-Prob>chi2 & 0.00 & & 0.00 & \\
\hline \multicolumn{5}{|c|}{ Average Marginal Effects } \\
\hline hpGDPgap & -2.056 & & -2.022 & \\
\hline ib & -.004 & & -0.006 & \\
\hline BEC & -.117 & & -0.343 & \\
\hline adj_type $=2$ & & & -0.079 & \\
\hline adj_type $=3$ & & & -0.118 & \\
\hline
\end{tabular}

Notes: - Cluster-robust standard errors are in parenthesis. - **, * indicate statistical significance at $5 \%$ and $10 \%$ levels, respectively. - Marginal effects $(\mathrm{dP} / \mathrm{dZ})$ indicate the marginal change in the probability of success. - In the estimation of the Differential Effect, Average Marginal Effects of "BEC" are calculated making the average of the Marginal Effects obtained considering only the obs. in the specific "id_dfbTYPE" category; while the same for "hpGDPgap" and "ib" are the average of the Marginal Effects obtained considering all the observations used for the estimation.

The estimation output shows a negative effect for all the three explanatory variables included, which is however not significant for the interest bill. By converting the coefficients in terms of Average Marginal Effects, it emerges that a 1\%-point increase of the GDP gap reduces by more than double the probability of observing a DGR reduction. The marginal effect of the GDP gap is surprising for its magnitude, but reasonable anyway. Indeed, when the gap increases, the deficit is likely to increase and this inflates the debt as a consequence. The interest bill turns out non-significant. Following the interpretation by Nickel et al. (2010), this might signal that the outstanding debt burden (as reflected by the interest bill) does not influence the DGR dynamics. 
As for the effect of fiscal consolidations (BEC), a 1\%-point increase reduces the probability of observing a DGR reduction by $12 \%$. Such adverse effect confirms what found through the comparison of distributions in section 5: fiscal consolidations are more likely associated with a 2-year cumulated DGR increase.

In relation to the probability of observing a DGR reduction estimated above, we want to check also whether or not a difference emerges between FCs based more on a tax-increase with respect to FCs based more on spending cuts. To distinguish such differential effect, we employ an ad-hoc categorical variable $A D J$ _TYPE $E_{i t}$ defined as follows:

$$
\text { ADJ_TYPE } E_{i t}=\left\{\begin{array}{l}
1 \text { if } \varphi>66 \% \\
2 \text { if } 33 \% \leq \varphi \leq 66 \% \\
3 \text { if } \varphi<33 \%
\end{array}\right.
$$

where $\varphi$ is the share of tax-increase over the total consolidation (to wit, $\varphi=25 \%$ means that only $25 \%$ of the budget consolidation is due to a tax-increase, while the remaining $75 \%$ is achieved through a spending cut). Then, $A D J_{-} T Y P E_{i t}=1$ signals a fiscal consolidation mainly tax-based, $A D J_{-} T Y P E_{i t}=3$ signals a fiscal consolidation mainly based on spending cuts, while $A D J_{-} T Y P E_{i t}=2$ a mixed fiscal consolidation. ${ }^{10}$

We interact the categorical $A D J J_{-} T Y P E_{i t}$ variable with the continuous $B E C_{i t}$ variable and include it in the estimation of eq.(12). ${ }^{11}$ Since we use as reference the tax-based group, we estimate the differential effect of savings-based consolidations and mixed consolidations with respect to tax-based consolidations. Results are in Table 5 - Column 2.

The estimation output shows that a difference indeed emerges between savings-based and mixed consolidations with respect to tax-based ones: tax-based consolidations are associated with a much lower probability of observing a major DGR reduction. In particular, a $1 \%$-point increase in tax-based consolidations decreases the probability of a observing a DGR reduction by $34.3 \%$, while such decrease is only $7.9 \%$ for mixed consolidations and $11.8 \%$ for savings-based consolidations. Then, tax-based consolidations seem to be by far more adverse. This finding is in line with many contributions which signal a more negative effect of tax-based FCs on the GDP (Alesina et al. 2013).

\footnotetext{
We consider also the mixed-consolidations category to separate more markedly the tax-based from the savingsbased group.

Then, three dummy variables, one for each category of $A D J$ TYPE, are multiplied by BEC. Results do not change significantly when we use directly the categorical variable $A D J$ TYPE without multiplying it for BEC

As for the result on the mixed-consolidations category, it may lead to believe that a good mix of spending-cuts and tax-increases is perhaps the best policy option. However, such statement should be based on further details about the consolidation which are not available in Devries et al. (2011)'s data set.
} 


\section{Concluding Remarks}

In this paper we have studied the effect of fiscal consolidations on the debt/GDP ratio evolution in a set of EU countries over the 1980-2009 period. Our results can be summarised as follows. Fiscal consolidations appear to have a favourable short-term effect, namely, they seem to temporary interrupt the growth tendency of the debt/GDP ratio. However, the medium-term effect results to be adverse. Indeed, fiscal consolidations are more likely associated with a 2-year cumulated DGR increase. This result is robust with respect to alternative analytical methods. Furthermore, the analysis shows that adverse medium-term outcomes are more likely when fiscal consolidations are based more on spending cuts than on an increase in taxation.

A difference between the short and medium-term effect therefore emerges in our analysis: the former is favourable, while the latter is generally adverse. A plausible explanation can be found in Guajardo et al. (2011) where the authors show that the effect of fiscal consolidations on real GDP achieves its peak within two years. Then, the different timing of the effect of fiscal consolidations on the deficit (in level) and on the output may explain the difference between the short and medium-term. This explanation is in line with other studies (among the others, Clinton et al. 2011) which highlight a varying effect of fiscal consolidations on macroeconomic variables over time. As for the less adverse effect of savings-based fiscal consolidations, this is not surprisingly. Indeed, many contributions have found the same and explain why those impact less the GDP (among the others, Alesina et al. 2013).

On the whole, the policy conclusion of this paper is that fiscal consolidations cannot be sold as effective ways to reduce the debt-to-GDP ratio; arguments based on short-term developments might be misleading about this. In case a fiscal consolidation is tempted anyway, savings-based ones should be preferred. 


\section{References}

Alesina A., C. Favero and F. Giavazzi (2013). "The output effect of fiscal consolidations", mimeo.

Alesina A. and S. Ardagna (2010). Large Changes in Fiscal Policy: Taxes versus Spending. In B. J. R., Tax Policy and the Economy, Vol 24 (Vol. 24). Cambridge MA: NBER.

Alesina A., R. Perotti, F. Giavazzi, and T. Kollintzas (1995). "Fiscal Expansions and Adjustments in OECD Countries", Economic Policy, 10(21): 205-248.

Cafiso G. (2012a). Debt Developments and Fiscal Adjustment in the EU. Intereconomics, Volume 47, Number 1.

Cafiso G. (2012b). A Guide to Public Debt Equations, November $2012-3^{\text {rd }}$ version, SSRN eLibrary.

Clinton K., M. Kumhof, D. Laxton, and S. Mursula (2011). "Deficit reduction: Short-term pain for long-term gain”, European Economic Review, 55:118-139.

Conen G., M. Mohr and R. Straub (2008). "Fiscal consolidation in the euro area: long run benefits and short-run costs", Economic Modelling, 25: 912-932.

Conover W. J. (1999). Practical Nonparametric Statistics. New York: Wiley.

Corsetti G., K. Kuester, A. Meier and J. Muller (2010). Debt consolidation and fiscal stabilization of deep recessions, American Economic Review, 100(2): 41-45.

Corsetti G., A. Meier and J. Muller (2012). Fiscal stimulus with spending reversals, Review of Economics and Statistics, 94: 878-895.

Cwik T. and V. Wieland (2011). "Keynesian government spending multipliers and spillovers in the euro area", Economic Policy, 26(67): 493-549.

Devries P., J. Guajardo, D. Leigh and A. Pescatori (2011). "A New Action-based Dataset of Fiscal Consolidation”, IMF Working Paper, International Monetary Fund.

Escolano J. (2010). "A Practical Guide to Public Debt Dynamics, Fiscal Sustainability, and Cyclical Adjustment of Budgetary Aggregates", IMF Technical Notes and Manuals, International Monetary Fund.

Everitt B. S., S. Landau and M. Leese (2001). Cluster Analysis. London: Arnold. 
Giavazzi F. and M. Pagano (1990). "Can Severe Fiscal Contractions Be Expansionary? Tales of Two Small European Countries". In O. J. Blanchard \& S. Fischer (Eds.), NBER Macroeconomics Annual 1990, MIT Press.

Gros, D. (2011). "Can Austerity be self-defeating?", Vox.

Guajardo J., D. Leigh and A. Pescatori (2011). "Expansionary Austerity: New International Evidence", IMF Working Paper, International Monetary Fund.

Krugman P. (2011). "Self-defeating Austerity", New York Times column, 7 July.

Nickel C., P. Rother and L. Zimmermann (2010). "Major Public Debt Reductions: Lessons from the past, lessons for the future", ECB Working Paper Series, No 1241.

Perotti R. (1996). "Fiscal consolidation in Europe: Composition matters", American Economic Review, 86(2): 105-110.

Perotti R. (2013). "The Austerity Myth: Gain without Pain?", in A. Alesina and F. Giavazzi (eds.) "Fiscal Policy After the Financial Crisis", NEBR.

Ramey V. (2013). "Government Spending and Private Activities" in A. Alesina and F. Giavazzi (eds.) "Fiscal Policy After the Financial Crisis", NEBR.

Romer C. D. and D. H. Romer (2010). "The Macroeconomic Effects of Tax Changes: Estimates Based on a New Measure of Fiscal Shocks", American Economic Review, 100(3): 763-801.

Sutherland D., P. Hoeller and R. Merola (2012). "Fiscal Consolidation: Part 1. How Much is Needed and How to Reduce Debt to a Prudent Level?", OECD Economics Department Working Papers, No. 932, OECD Publishing.

Stata (2009). Stata Base Reference Manual, release 11. Stat Corp, College Station, Texas.

Wilcoxon F. (1945). "Individual comparisons by ranking methods", Biometrics, 1: 80-83. 


\section{Appendix I - Country Groups}

In this appendix we develop a cluster analysis which splits our country sample into two groups. The aim is to understand which countries exhibit a similar DGR dynamics in case of fiscal consolidations.

Cluster analysis attempts to determine the natural groupings (or clusters) of observations (Everitt et al. 2001). It breaks the observations into $k$ distinct number of non-overlapping groups; in our application $k=2$. We implement the "means" partition method. ${ }^{13}$ The cluster analysis is based on $\Delta_{2} b_{i t+1}$ values only in yes-FC years because we want to cluster countries for their different response to a FC event.

The cluster analysis generates 2 clusters (A and B); we label as "cluster A" the one with the lower $\Delta_{2} b_{i t+1}$ mean value (smaller 2-year cumulated change). Since we have multiple time observations per country, it happens that some observations of a country fall in cluster $A$, while others in the cluster B. We denote as non-virtuous the countries for which the portion of "cluster A" observations is below the $25^{\text {th }}$ percentile of the all-countries distribution of "cluster A" portions. Some details and the final result of the present cluster analysis are reported in Table 6.

Table 6. Cluster Analysis output

\begin{tabular}{c|c|c|c|c|c|c|c|c|c|c}
\hline \hline Country & $\begin{array}{c}\text { \# of } \\
\text { FC }\end{array}$ & $\begin{array}{c}\text { \# obs in } \\
\text { cluster A }\end{array}$ & $\begin{array}{c}\text { \% obs in } \\
\text { cluster A }\end{array}$ & $\begin{array}{c}\text { Final } \\
\text { group }\end{array}$ & Country & $\begin{array}{c}\text { \# of } \\
\text { FC }\end{array}$ & $\begin{array}{c}\text { \# obs in } \\
\text { cluster A }\end{array}$ & $\begin{array}{c}\text { \% obs in } \\
\text { cluster A }\end{array}$ & $\begin{array}{c}\text { Final } \\
\text { group }\end{array}$ \\
\hline AT & 5 & 4 & .80 & V & IE & 6 & 2 & .33 & NV \\
\hline BE & 10 & 5 & .50 & V & IT & 12 & 8 & .67 & V \\
\hline DE & 15 & 9 & .60 & V & NL & 3 & 3 & 1.00 & V \\
\hline DK & 5 & 4 & .80 & V & PT & 7 & 1 & .14 & NV \\
\hline ES & 10 & 4 & .40 & NV & SE & 7 & 6 & .86 & V \\
\hline FI & 6 & 4 & .67 & V & UK & 6 & 5 & .83 & V \\
\hline FR & 9 & 6 & .67 & V & & & & & \\
\hline \hline
\end{tabular}

Note: The Table reports the number of fiscal consolidation episodes for each country, the number of observations falling in cluster A and the portion over the total. In the "final group" column, V stays for "virtuous" and NV for "non-virtuous", as explained in text.

\footnotetext{
${ }^{13}$ In means-clustering, each observation is assigned to the group whose mean is closest, and then based on that categorization, new group means are determined. These steps continue until no observations change groups. The algorithm begins with $\mathrm{k}$ seed values, which act as the $k$ group means. There are many ways to specify the beginning seed values. We specify that $k$ partitions are formed randomly among the observations to be clustered, then the group means from the $\mathrm{k}$ groups defined by this partitioning are used as the starting group centres. As similarity measure we use the Euclidean distance.
} 
Based on this criterion, the non-virtuous group consists of Spain (ES), Ireland (IE) and Portugal (PT); all the other countries are in the virtuous group. The same countries are assigned to the non-virtuous group when the criterion chosen is that the percentage of observations in cluster $\mathrm{A}$ is below $50 \%$. 


\section{Appendix II - Consecutive Fiscal Consolidations}

Fiscal consolidations (FCs) in Devries et al. (2011)'s data set are mainly definitive. To wit, only in 8 cases over 112 available a FC is followed by a correction. At the same time, there are many multiyear periods when consecutive FCs are observed in a country. Consecutive FCs are interesting to look at because they resemble what credible plans for the DGR reduction should be.

Ex-ante, it is difficult to foresee what the GDP response is going to be during such multiyear periods and, consequently, what the final effect on the DGR evolution will be. In Table 7 we report the 18 cases when there are, at least, three consecutive years of $F C$ in a row. Germany is the country which has enforced more consecutive FCs, while the longest FC periods are for Italy (1991-1998) and the Netherlands (1981-1988).

Let us now consider the cumulated change from the year after the last of FC to the year before the first of FC: $\Delta_{m} b_{t+m}=b_{t+m}-b_{t-1}$, where $t$ is the first year of the FC period and $m$ is the number of FC years starting in $t$. The values of $\Delta_{m} b_{t+m}$ are reported in Table 7 . When we look at such cumulated change (only values above $1 \%$ GDP), we count 13 periods when the DGR increased and only 3 when it decreased over a total of 16 observations available. As for the correlation between the total budget effect of the consecutive FCs (column "tot BEC" in Table 7) and the cumulated change (column " $\Delta_{m} b_{t+m}$ " in Table 7), this is shown in Figure 3. In general, larger FCs are associated with larger $\Delta_{m} b_{t+m}$, but a strong relationship does not emerge.

On the whole, the consideration of multiyear consolidation strategies does not seem to alter the main findings of our analysis. Indeed, also consecutive fiscal consolidations seem to be associated with positive cumulated DGR variations. 
Table 7. Consecutive FC periods

\begin{tabular}{cccccc}
\hline \hline country & Start & End & $\mathrm{N}^{\circ}$ years & tot BEC & $\Delta_{\mathrm{m}} \mathbf{b}_{\mathrm{t}+\mathrm{m}}$ \\
\hline be & 1982 & 1985 & $(4)$ & 5.75 & 35.20 \\
& 1992 & 1994 & $(3)$ & 3.86 & 3.26 \\
\hline $\mathrm{dk}$ & 1983 & 1986 & $(4)$ & 5.97 & -2.10 \\
\hline $\mathrm{fi}$ & 1992 & 1997 & $(7)$ & 11.43 & 26.10 \\
$\mathrm{fr}$ & 1995 & 1997 & $(3)$ & 2.11 & 10.07 \\
\hline $\mathrm{de}$ & 1982 & 1984 & $(3)$ & 2.23 & 5.9 \\
& 1991 & 1995 & $(5)$ & 3.67 & 16.13 \\
& 1997 & 2000 & $(4)$ & 2.50 & 0.40 \\
\hline ie & 1982 & 1988 & $(7)$ & 10.05 & 24.40 \\
\hline it & 1991 & 1998 & $(8)$ & 19.23 & 19.05 \\
& 2004 & 2007 & $(4)$ & 4.72 & 1.89 \\
\hline $\mathrm{nl}$ & 1981 & 1988 & $(8)$ & 12.98 & $\mathrm{n} . \mathrm{a}$. \\
& 1991 & 1993 & $(3)$ & 1.73 & -1.10 \\
\hline $\mathrm{pt}$ & 2005 & 2007 & $(3)$ & 3.65 & 13.93 \\
\hline es & 1992 & 1997 & $(6)$ & 6.64 & 20.71 \\
\hline se & 1993 & 1998 & $(6)$ & 10.6 & 1.11 \\
\hline uk & 1980 & 1982 & $(3)$ & 2.19 & 11.04 \\
& 1994 & 1999 & $(6)$ & 2.62 & -3.51 \\
\hline \hline
\end{tabular}

Figure 3. Relation between totBEC and $\Delta_{m} b_{t+m}$

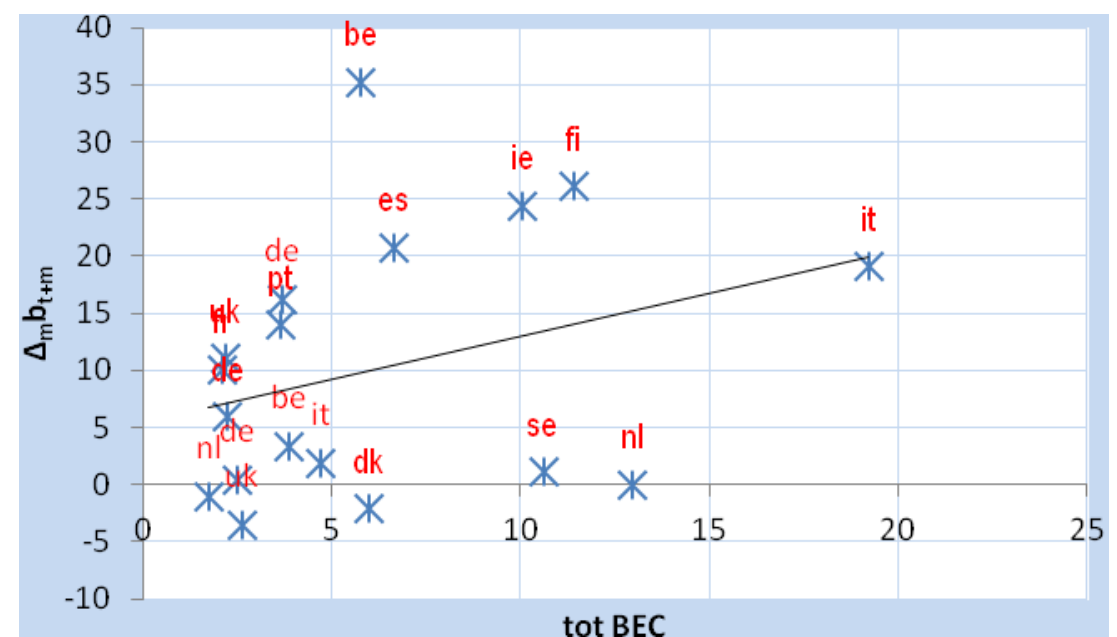

